\title{
A MONOLITHIC EWOD CHIP BY SURFACE MICROMACHINING
}

\author{
Wyatt C. Nelson* and Chang-Jin "CJ" Kim
}

Mechanical and Aerospace Engineering Department, University of California, Los Angeles, (UCLA)

\begin{abstract}
We present a surface micromachining process to build monolithic two-plate electrowetting-on-dielectric (EWOD) droplet microfluidic chips. Our fabrication method removes the need for device alignment and assembly, and provides excellent control of cavity heights, which can be as small as a few microns. The monolithic EWOD chip is evaluated by performing creation, transport, splitting, and merging of $100 \mathrm{pL}$ water droplets immersed in silicone oil.
\end{abstract}

\section{INTRODUCTION}

Evolved from device ideas implementing conventional electrowetting [1], almost all electrowetting devices of today use a thin dielectric layer on surface electrodes, thus called electrowetting-on-dielectric (EWOD) [2; 3]. The two-plate configuration (Figure 1) is most often used in lieu of a single planar chip in order to facilitate droplet creation and splitting even in air (vs. in oil) [4]. While it is possible to draw random liquid branches from a sessile droplet using the electrowetting force [5] or to transport sessile droplets on one-plate EWOD devices on coplanar electrodes with the droplet electrically floating [6] or grounded-from-below [7], facile creation and splitting has not been demonstrated on a one-plate device without the use of additional forces such as dielectrophoresis [8]. Squeezing the droplet between hydrophobic parallel plates into a disk-like shape increases its internal pressure. In this state the dominant radius of curvature is related to the cavity height rather than the droplet radius. Under this condition, the contact angle change due to electrowetting can generate pressures sufficient for splitting. [4;9]

A common way to produce a two-plate EWOD chip involves separate fabrication of the bottom substrate and top plate, followed by alignment, assembly, and bonding. The typical EWOD substrate is a glass or silicon wafer with photolithographically patterned surface electrodes covered by a thin dielectric, e.g. silicon dioxide or parylene. The typical top plate is ITO-coated glass to allow for visual inspection and electrical grounding. Both plates are coated with a spin-on hydrophobic fluoropolymer such as Teflon ${ }^{\circledR}$ or Cytop ${ }^{\circledR}$ prior to assembly. A variety of materials are used for spacing and/or bonding, including double-sided adhesive tape, SU-8, and rubber gaskets. With the exception of SU-8, spacers are manually aligned before the top plate is aligned and bonded. These post-processing steps make it challenging for the user to create a two-plate chip with precise cavity dimensions, especially at the micron scale. Additionally, top plate alignment and bonding complicates batch fabrication and integration of other fluidic and electrical components.

Several innovative alternatives to traditional cleanroom microfabrication have been reported for building EWOD substrates, including post-processed printed circuit boards [10], and a rapid prototyping technique in which electrodes are patterned by inkjet printing onto flexible copper-coated polyimide substrates [11]. Currently, though, no fabrication process has been reported to yield monolithic two-plate EWOD chips.

The monolithic chip presented here alleviates many of the limitations associated with assembled devices. For example, in the two-plate scenario the minimum droplet size that can be split by the electrowetting force is directly related to the cavity height, e.g. the cavity height should be less than about 1/5 the droplet radius [4,9], and therefore manipulation of picoliter droplets requires micron cavity heights, which are easy to achieve using our proposed surface micromachining process. Reports of creating and splitting picoliter droplets in two-plate EWOD chips [12; 13] have demonstrated downscaling of the typical nanoliter devices by simply using more precise spacers and more careful assembly techniques in order to achieve $\sim 10$ micron cavity heights. The next-generation monolithic device, however, will accurately create pico- and femtoliter droplets without any assembly necessary, opening the door to more complex systems, such as pressurized processing, chip stacking, and integration with other electronic or fluidic devices.

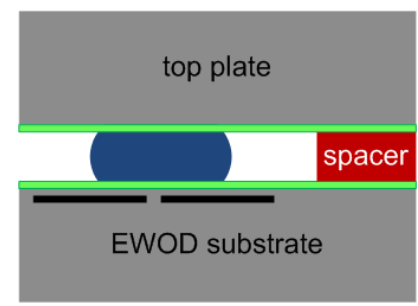

(a) assembled EWOD surface micromachined enclosure with electrodes

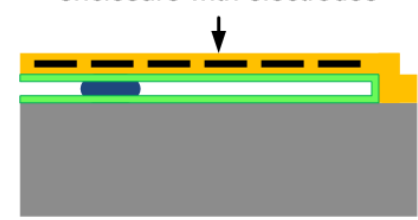

(b) monolithic EWOD
Figure 1: (a) The typical two-plate device consists of a top plate bonded by a spacer to an EWOD substrate, which has patterned electrodes and a dielectric layer. (b)The monolithic device has a thin-film enclosure embedded with EWOD electrodes. In both (a) and (b), working inner surfaces are coated hydrophobic.

\section{CHIP FABRICATION}

\section{Materials selection}

The characteristics of the inner cavity surfaces are important to EWOD performance for several reasons, which guided our choice to use the silicon device layer of a SOI wafer to serve as a sacrificial mold for the cavity. First, the device layer is extremely smooth, minimizing the number of defects that will exist in the membrane layer upon release. In most cases, surface roughness and pinhole defects are hindrances to EWOD droplet actuation and may prevent movement of the contact line altogether. Second, the device layer enables excellent control of cavity height because it can be ordered to have a uniform thickness ranging from less than a micron to over one hundred microns. Such precise control of cavity height enables precious control of droplet volumes. Finally, silicon is an excellent sacrificial material for this application because it can be removed by $\mathrm{XeF}_{2}$, which is clean and highly selective to the dielectrics silicon dioxide and silicon nitride.

The four micron thick surface micromachined membrane, which serves as the top plate, is about 100 times thinner than plates used for assembled chips. By using a special low-stress silicon nitride grown by plasma-enhanced chemical vapor deposition (PECVD), it was possible to span over three hundred microns without warping or buckling. In fact, the nitride membranes reported here, which are suspended 10 microns above the substrate, were subject to wet photoresist stripping and repeating rinsing steps upon release, with no stiction.

A key requirement for EWOD actuation of aqueous liquids is that the dielectric surface must be sufficiently hydrophobic; otherwise the droplet will not slide. For the typical case of coating a flat, open surface hydrophobic, spin-coating a fluoropolymer 
solution is straightforward, and the deposited thickness is easy to measure, e.g. by profilometry. Coating the inner surfaces of a 10 $\mu \mathrm{m}$ cavity, however, is somewhat more complicated. Our current method is to fill the cavity with fluoropolymer solution and spin the chip at a very high speed $(7000 \mathrm{rpm})$ so that the liquid flows out of the cavity via release openings. While we observed through electrowetting experiments that this technique can yield hydrophobic inner surfaces, we have not characterized the resultant film thickness or uniformity. In some cases, we observed thick polymer residues where membrane anchors had obstructed the coating flow. We are currently developing a more controlled coating technique that will be far less sensitive to the chip layout and cavity height.

\section{Fabrication process flow}

A SOI wafer was used as the substrate for the monolithic chip. Figure 2 shows the process flow, which begins with deep reactive ion etching (DRIE) of the $10 \mu \mathrm{m}$ silicon device layer using photoresist (AZ5214) as a mask. The device layer serves as a mold for the eventual thin-film membrane and thus determines the cavity height. Next, a $100 \mathrm{~nm}$ thermal oxide is grown on the etched device layer. This layer was found to be necessary as an etch stop defining the inner top surface of the cavity during the $\mathrm{XeF}_{2}$ release etch, which showed poor selectivity to the low-stress silicon nitride that was subsequently grown by PECVD. This nitride and the underlying oxide form a composite insulating layer which sustains the electric field necessary to generate EWOD force.

Metal electrodes, composed of $200 \mathrm{~nm}$ thick gold upon a 5 $\mathrm{nm}$ titanium or chromium adhesion layer, are deposited by e-beam evaporation and wet etched. When it was desirable to have transparent electrodes, the gold was completely etched away from the device area, leaving only the highly transparent titanium layer, through which droplets can be seen. The membrane is completed by another PECVD low-stress nitride, which is then etched by RIE to form release openings. Importantly, the photoresist mask used to pattern the release openings is also used to protect the top surface of the membrane during the $\mathrm{XeF}_{2}$ release etch, which exhibited selectivities of less than 1:20 to our low-stress nitride.

As shown in Figure 2, the $\mathrm{XeF}_{2}$ first removes the exposed silicon, and then continues to etch the sacrificial silicon under the enclosure and between the anchors to completely evacuate the cavity. Upon release, the chips are stripped of photoresist using ALEG 355 at $100^{\circ} \mathrm{C}$ and thoroughly rinsed in DI water. A drop of 6 wt $\%$ solution of Cytop ${ }^{\circledR}$ is then placed onto chip, which it spontaneously wets, filling up the cavity. The chip is then affixed to a carrier wafer, which is spun at $7000 \mathrm{rpm}$ for 2 minutes to ensure that most of the fluoropolymer solution flows out of the cavity via the release openings. Finally, the chip is baked on a hot plate at $200{ }^{\circ} \mathrm{C}$ for ten minutes to ensure that the solvent is completely evaporated.

\section{Chip layout}

The monolithic chip layout is composed of three basic elements: anchors, electrodes, and release openings. Figure 2 illustrates a very basic layout, and Figure 3 a shows the layout of an actual chip that was fabricated using the process of Figure 2 used to demonstrate EWOD. In our experiments, liquids were pumped into the cavity manually by a syringe. To establish a fluid connection, a NanoPort ${ }^{\circledR}$ was placed over the inlet, located several millimeters away from the EWOD electrodes.

Regions of the membrane in which there are embedded EWOD actuation electrodes span several hundred microns. To measure membrane flatness, a profilometer scan was performed along the dashed line path shown in Figure 3a. The profile, plotted in Figure 3b, indicate a maximum deflection of about $200 \mathrm{~nm}$, resulting in an approximate slope of 0.0013 from the edge to the center of the cavity, or a $1.3 \%$ error associated with the height of a $100 \mathrm{pL}$ droplet in this region.
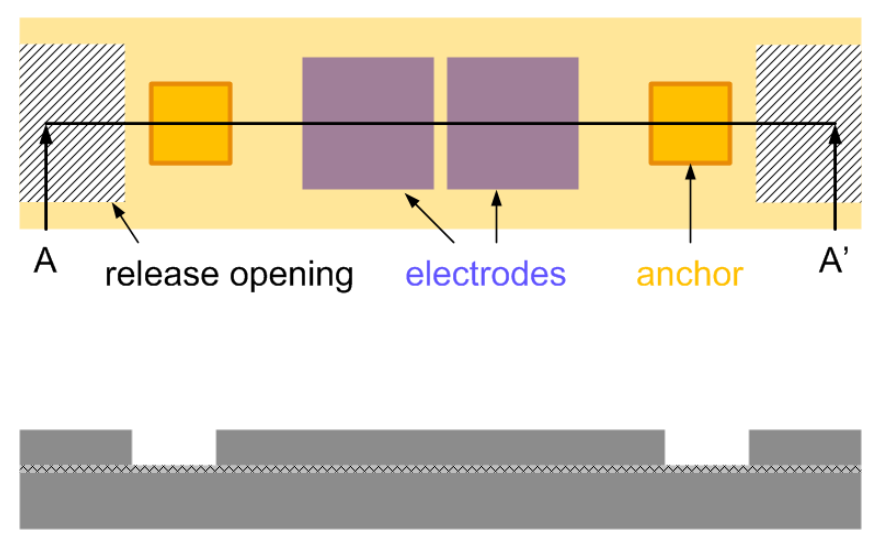

1. DRIE device layer $(10 \mu \mathrm{m})$ of SOI wafer

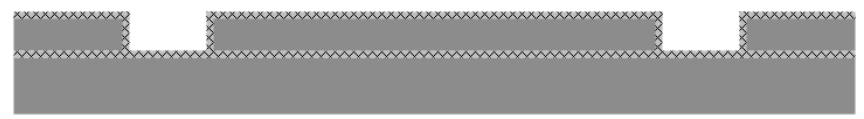

2. Thermal oxidation $(100 \mathrm{~nm})$

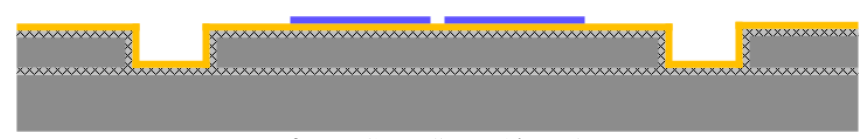

3. PECVD $\operatorname{Si}_{x} N_{y}(1 \mu \mathrm{m})$

4. Evaporate \& pattern Ti/Au $(5 / 100 \mathrm{~nm})$

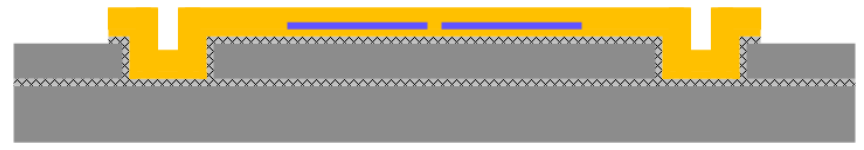

5. PECVD Si $N_{x}(3 \mu \mathrm{m})$

6. RIE etch release holes

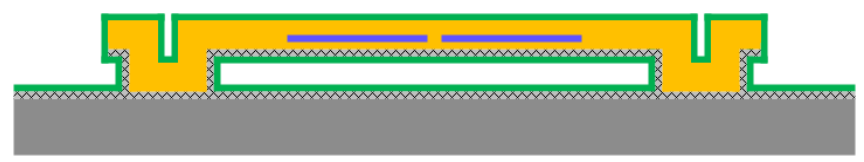

7. $\mathrm{XeF}_{2}$ release etch

8. Spin-coat hydrophobic polymer

$\mathrm{Si} \mathrm{SiO}_{2}$ $\mathrm{Si}_{\mathrm{x}} \mathrm{N}_{\mathrm{y}}$ $\mathrm{Ti} / \mathrm{Au}$ Cytop ${ }^{\mathbb{1}}$

Figure 2: Top view of basic layout for enclosed EWOD devices, and the process flow at cross section $A-A$ '. The process begins with DRIE through the device layer of an SOI wafer having $200 \mathrm{~nm}$ buried oxide. 


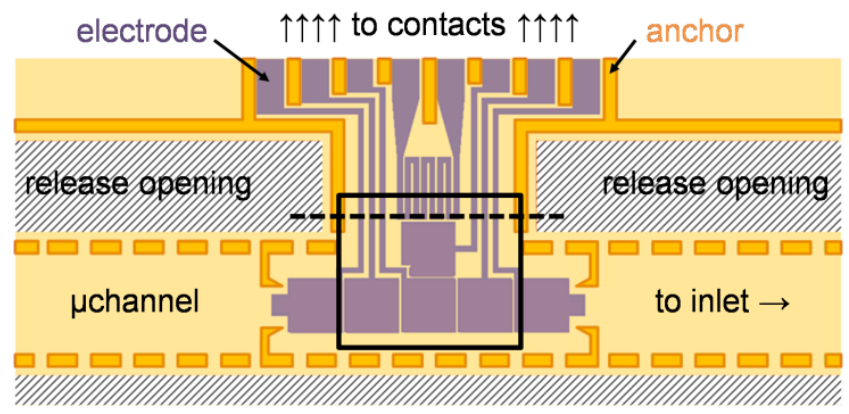

(a) device layout

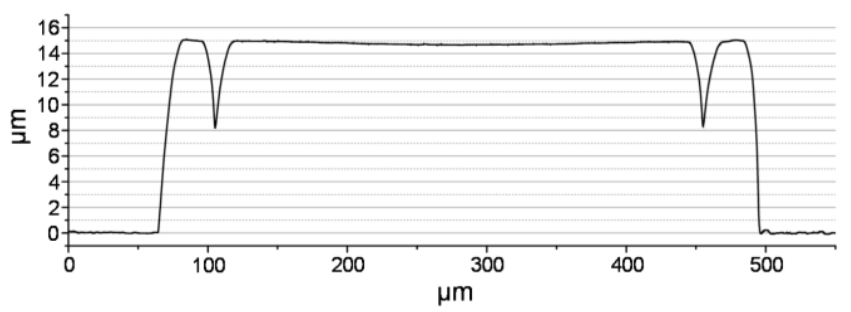

(b) Profilometer scan along dashed line

Figure 3: (a) device layout (black box indicates field of view in Figure 4) and (b) a profilometer scan along the path indicated by the dashed line in (a). Note that the profilometer stylus is too large to capture the shape of the $20 \mu \mathrm{m}$ wide anchors.

\section{RESULTS AND DISCUSSION}

Droplet creation, transport, splitting, and merging were performed in order to evaluate the chip. Figure 4, for which the field of view corresponds to the black box in Figure 3, shows video frames of creation and transport (a.1 to a.6) and splitting and merging (b.1 to b.4). Actuations of DI water in silicone oil (0.6 cSt) were achieved by applying driving voltages $36 \mathrm{~V}_{\mathrm{rms}}$ at $100 \mathrm{~Hz}$.

Figure $4 \mathrm{a} .1$ provides the initial conditions prior to EWOD actuation and indicates the water-oil interface which separates the water reservoir from the oil medium. The water reservoir is in contact with anchors above the field of view. The faint grey $100 \mathrm{x}$ $100 \mu \mathrm{m}$ electrodes are made of avery thin ( $5 \mathrm{~nm}$ thick) titanium to ensure that the water-oil interface can be clearly seen. During actuations, biased electrodes are labeled "ON" and all other electrodes as well as the silicon substrate were grounded. Frames a. 2 to a. 4 depict the liquid extension process in which a small volume of water is pulled away from the reservoir, but still connected by a fluid neck. After the droplet is about three electrode lengths away, the neck breaks and a droplet is created (a.5) and transported (a.6).

Splitting and merging is shown by the series of video frames in Figure 4b. A parent droplet is pulled in opposite directions by EWOD (b.2), producing two daughter droplets having similar volumes (b.3), which are merged by biasing the central electrode (b.4).

Water droplets were actuated in an oil medium in order to avoid evaporation. Evaporation plays a much greater effect in this scale, which is $\sim 10$ times smaller than the usual EWOD devices of today having $\sim 1 \mathrm{~mm}$ pad size. Another consequence of using oil instead of air as the filler medium in any EWOD chip is that the
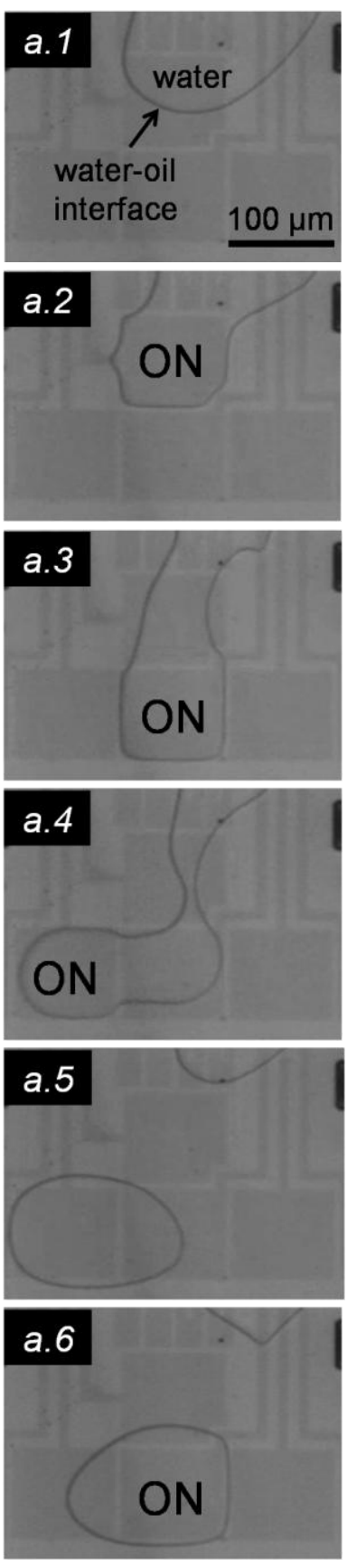

Figure 4: (a) Video frames of droplet creation and transport. (b) Video frames of droplet splitting and merging. The driving voltage was $36 V_{\text {rms }}$ at $100 \mathrm{~Hz}$

actuation voltage is relatively low as a result of reduced hysteresis drag [14]. In many applications, though, oil-free operation is preferred and can be achieved via humidity control.

\section{CONCLUSIONS}

We have developed a surface micromachined monolithic twoplate EWOD chip and used it to demonstrate creation, transport, splitting, and merging of $100 \mathrm{pL}$ water droplets in a $10 \mu \mathrm{m}$ high oil-filled cavity. Many of the limitations associated with alignment and bonding of conventional two-plate EWOD chips are bypassed through this new fabrication process, which holds great potential for producing highly integrated, picoliter digital microfluidic systems. 


\section{ACKNOWLEDGEMENTS}

The authors would like to thank Dr. Prosenjit Sen and all members of the UCLA Micro and Nano Manufacturing Laboratory for participating in helpful discussions. For this work WN was supported by the NIH (R01 RR020070) and the NSF Graduate Research Fellowship Program (GRFP) and an NSF Integrative Graduate Education and Research Traineeship (DGE-0654431) through the UCLA Materials Creation Training Program (MCTP).

\section{REFERENCES}

[1] E. Colgate, H. Matsumoto, "An investigation of electrowetting-based actuation," J. Vac. Sci. Technol. A, 8, 4, pp 3625-3633 (1990).

[2] J. Lee, H. Moon, J. Fowler, T. Schoellhammer, and C.-J. Kim, "Electrowetting and electrowetting-on-dielectric for microscale liquid handling," Sens. Actuators, A, 95, pp 259268 (2002).

[3] H. Moon, S. K. Cho, R. L. Garrell, and C.-J. Kim, "Low voltage electrowetting-on-dielectric," J. Appl. Phys., 92, 7, pp 4080-4087 (2002).

[4] S. K. Cho, H. Moon, and C.-J. Kim, "Creating, Transporting, Cutting, and Merging Liquid Droplets by ElectrowettingBased Actuation for Digital Microfluidic Circuits," $J$. Microelectromech. Syst., 12, 70 (2003).

[5] F. Mugele, S. Herminghaus, "Electrostatic stabilization of fluid microstructures," App. Phys. Lett., 81, 12, pp 2303-2305 (2002).

[6] U.-C. Yi and C.-J. Kim, "Characterization of electrowetting actuation on addressable single-side coplanar electrodes," $J$. Micromech. Microeng., 16, pp 2053-2059 (2006).
[7] C.G. Cooney, C.-Y. Chen, M.R. Emerling, A Nadim, and J.D. Sterling, "Electrowetting droplet microfluidics on a single planar surface," Microfluid. Nanofluid., 2, pp 435-446 (2006).

[8] T.B. Jones, M. Gunji, M. Washizu, and M.J. Feldman, "Dielectrophoretic liquid actuation and nanodroplet formation," J. Appl. Phys., 89, pp 1441-1448 (2001).

[9] H. Ren, "Electrowetting-based sample preparation: an initial study for droplet transportation, creation and on-chip digital dilution," Ph.D. Thesis, Duke University (2004).

[10] J. Gong and C.-J. Kim, "Direct-Referencing TwoDimensional-Array Digital Microfluidics Using Multi-Layer Printed Circuit Board," J. Microelectromech. Syst., Vol. 17, 2008, pp. 257-264.

[11] M. Abdelgawad and A. R. Wheeler, "Rapid Prototyping in Copper Substrates for Digital Microfluidics," Adv. Mater., 19 pp 133-137 (2007)

[12] S. U. Son, D. Chatterjee, and R. L. Garrell, "Electricallyinduced splitting and generation of sub-nanoliter droplets in air on a digital microfluidic device," Proc. Int. Conf. $\mu$ TAS 2006, pp 128-130.

[13] J. H. Song, R. Evans, Y.-Y. Lin, B.-N. Hsu, and R. B. Fair, “A scaling model for electrowetting-on-dielectric microfluidic actuators," Microfluid. Nanofluid., 7, 75 (2009).

[14] M. G. Pollack, A. Shenderov, and R. B. Fair, "Electrowetting - based actuation of liquid droplets for microfluidic applications," Appl. Phys. Lett., 77, pp 17251727 (2000).

\section{CONTACT}

*W.C.Nelson, tel: +1-310-883-8971; wyattnelson@UCLA.edu 